\title{
Probes of the Standard Model effective field theory extended with a right-handed neutrino
}

\author{
Julien Alcaide, ${ }^{a, b}$ Shankha Banerjee, ${ }^{b}$ Mikael Chala ${ }^{b, c}$ and Arsenii Titov ${ }^{b}$ \\ ${ }^{a}$ Departament de Física Teòrica, Universitat de València and IFIC, Universitat de València-CSIC, \\ Dr. Moliner 50, E-46100 Burjassot, València, Spain \\ ${ }^{b}$ Institute for Particle Physics Phenomenology, Department of Physics, Durham University, \\ South Road, Durham DH1 3LE, U.K. \\ ${ }^{c}$ CAFPE and Departamento de Física Teórica y del Cosmos, \\ Universidad de Granada, E-18071 Granada, Spain \\ E-mail: julien.alcaide@uv.es, shankha.banerjee@durham.ac.uk, \\ mikael.chala@durham.ac.uk, arsenii.titov@durham.ac.uk
}

ABSTRACT: If neutrinos are Dirac particles and, as suggested by the so far null LHC results, any new physics lies at energies well above the electroweak scale, the Standard Model effective field theory has to be extended with operators involving the right-handed neutrinos. In this paper, we study this effective field theory and set constraints on the different dimension-six interactions. To that aim, we use LHC searches for associated production of light (and tau) leptons with missing energy, monojet searches, as well as pion and tau decays. Our bounds are generally above the $\mathrm{TeV}$ for order one couplings. One particular exception is given by operators involving top quarks. These provide new signals in top decays not yet studied at colliders. Thus, we also design an LHC analysis to explore these signatures in the $t \bar{t}$ production. Our results are also valid if the right-handed neutrinos are Majorana and long-lived.

Keywords: Beyond Standard Model, Effective Field Theories, Neutrino Physics ArXiv EPRINT: 1905.11375 


\section{Contents}

1 Introduction 1

2 Formalism $\quad 3$

2.1 Searches for one lepton and missing energy at the LHC 3

2.2 Monojet searches at the LHC 5

2.3 Pion decays 5

2.4 Tau decays 6

$\begin{array}{lll}2.5 & \text { Top decays } & 7\end{array}$

$\begin{array}{lll}3 & \text { Global constraints } & 9\end{array}$

$4 t \rightarrow b \ell N$ at the LHC $\quad 10$

5 Conclusions 14

\section{Introduction}

Despite its impressive success in describing particle physics phenomena across a wide range of energies, the Standard Model (SM) is known to be incomplete. The main experimental indication of new physics is the non-vanishing neutrino masses. On the theory side, the hierarchy problem, the flavour puzzle and the unification of couplings among others, also suggest the existence of particles beyond the SM. In light of the null results at the Large Hadron Collider (LHC), new particles might lie at energies, $\Lambda$, well above the electroweak (EW) scale, established by the Higgs vacuum expectation value (VEV), $v \sim 246 \mathrm{GeV}$.

For such scenarios, new physics can be described by the SM effective field theory (SMEFT) [1]. This theory extends the renormalisable SM Lagrangian with a tower of effective operators respecting the SM gauge symmetries, but not necessarily the global (accidental) ones. Within this framework, neutrinos are predicted to be Majorana, their mass being $m_{\nu} \sim \alpha_{5} v^{2} / \Lambda$ with $\alpha_{5}$ being the Wilson coefficient of the Weinberg operator [2]. Up to flavour indices, the latter can be considered to be the only independent effective operator of dimension five. At dimension six, this number goes up to 59 [3], assuming baryon number conservation. However, if neutrinos are Dirac particles, the SMEFT has to be extended with effective operators containing the right-handed $(\mathrm{RH})$ neutrino, $N$.

This extended EFT, known as $\nu$ SMEFT, was first considered in ref. [4], where also a complete set of operators in the $\nu$ SMEFT to dimension six was worked out. (See also ref. [5] for dimension five and ref. [6] for dimension seven.) This set was shown to be redundant in ref. [7], where an actual basis was provided. It is shown in table 1 for completeness. In this paper, we set constraints on these operators upon using searches for one lepton 


\begin{tabular}{|c|c|c|c|c|}
\hline \multirow{4}{*}{ 至 } & Operator & Notation & Operator & Notation \\
\hline & $\left(\overline{l_{L}} N\right) \tilde{H}\left(H^{\dagger} H\right)$ & $\mathcal{O}_{l N H}$ (+h.c.) & & \\
\hline & $\left(\bar{N} \gamma^{\mu} N\right)\left(H^{\dagger} i \overleftrightarrow{D_{\mu}} H\right)$ & $\mathcal{O}_{H N}$ & $\left(\bar{N} \gamma^{\mu} e_{R}\right)\left(\tilde{H}^{\dagger} i D_{\mu} H\right)$ & $\mathcal{O}_{H N e}$ (+h.c.) \\
\hline & $\left(\overline{l_{L}} \sigma_{\mu \nu} N\right) \tilde{H} B^{\mu \nu}$ & $\mathcal{O}_{N B}(+$ h.c. $)$ & $\left(\overline{l_{L}} \sigma_{\mu \nu} N\right) \sigma_{I} \tilde{H} W^{I \mu \nu}$ & $\mathcal{O}_{N W}$ (+h.c. $)$ \\
\hline \multirow{3}{*}{ 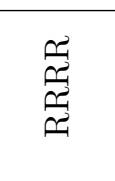 } & $\left(\bar{N} \gamma_{\mu} N\right)\left(\bar{N} \gamma^{\mu} N\right)$ & $\mathcal{O}_{N N}$ & & \\
\hline & $\left(\overline{e_{R}} \gamma_{\mu} e_{R}\right)\left(\bar{N} \gamma^{\mu} N\right)$ & $\mathcal{O}_{e N}$ & $\left(\overline{u_{R}} \gamma_{\mu} u_{R}\right)\left(\bar{N} \gamma^{\mu} N\right)$ & $\mathcal{O}_{u N}$ \\
\hline & $\left(\overline{d_{R}} \gamma_{\mu} d_{R}\right)\left(\bar{N} \gamma^{\mu} N\right)$ & $\mathcal{O}_{d N}$ & $\left(\overline{d_{R}} \gamma_{\mu} u_{R}\right)\left(\bar{N} \gamma^{\mu} e_{R}\right)$ & $\mathcal{O}_{d u N e}(+$ h.c. $)$ \\
\hline LLRR & $\left(\overline{l_{L}} \gamma_{\mu} l_{L}\right)\left(\bar{N} \gamma^{\mu} N\right)$ & $\mathcal{O}_{l N}$ & $\left(\overline{q_{L}} \gamma_{\mu} q_{L}\right)\left(\bar{N} \gamma^{\mu} N\right)$ & $\mathcal{O}_{q N}$ \\
\hline \multirow{2}{*}{ 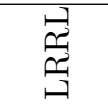 } & $\overline{\left(\overline{l_{L}} N\right) \epsilon\left(\overline{l_{L}} e_{R}\right)}$ & $\mathcal{O}_{l N l e}(+$ h.c. $)$ & $\overline{\left(\overline{l_{L}} N\right) \epsilon\left(\overline{q_{L}} d_{R}\right)}$ & $\overline{\mathcal{O}_{l N q d} \text { (+h.c.) }}$ \\
\hline & $\left(\overline{l_{L}} d_{R}\right) \epsilon\left(\overline{q_{L}} N\right)$ & $\mathcal{O}_{l d q N}$ (+h.c.) & $\left(\overline{q_{L}} u_{R}\right)\left(\bar{N} l_{L}\right)$ & $\mathcal{O}_{q u N l}$ (+h.c.) \\
\hline
\end{tabular}

Table 1. Basis of lepton and baryon number conserving dimension-six operators containing a RH neutrino $N$ [7]. $l_{L}$ and $q_{L}$ stand for the left-handed lepton and quark doublets, respectively. Likewise, $e_{R}$ and $u_{R}$ and $d_{R}$ stand for the right-handed leptons and the up and down quarks, respectively. We use the symbol $H$ to denote the Higgs doublet, while $\tilde{H}=\epsilon H^{*}$, where $\epsilon$ is the fully antisymmetric tensor in two dimensions. $B_{\mu \nu}$ and $W_{\mu \nu}^{I}$ represent the weak field strength tensors. Flavour indices are not shown explicitly.

$(e, \mu, \tau)$ and missing energy at the LHC, monojet searches at the LHC as well as pion and tau decays. For those operators not yet constrained by these observables, most of them involving top quarks, we suggest a novel search strategy based on a new rare top decay at the LHC.

A comment about cosmological constraints is in order. It is well known that in the absence of any other interactions, sterile neutrinos do not contribute to the number of relativistic species, $N_{\text {eff. }}$ This is no longer true in the $\nu$ SMEFT with a low cutoff $\Lambda$. Indeed, the interaction rate for producing $\mathrm{RH}$ neutrinos out of the thermal bath behaves as $\Gamma \sim$ $T^{5} / \Lambda^{4}$. The latter are in thermal equilibrium at the time of the Big Bang Nucleosynthesis (BBN) provided $\Gamma \gtrsim T_{\mathrm{BBN}}^{2} / m_{P}$, with $m_{P}$ being the Planck mass and $T_{\mathrm{BBN}} \sim \mathrm{MeV}$; namely if $\Lambda \lesssim 200 \mathrm{GeV} \sim v$. (There is no experimental evidence of the pre BBN era - some models even require reheating right before $T \sim \mathrm{MeV}[8,9]$ - so there are no actual cosmological constraints on the $\nu \mathrm{SMEFT}$ if $N$ decouples before BBN.)

Finally, $N$ could be also a very light Majorana neutrino with mass $m_{N}$. Even in the standard cosmological history, the contribution of such particle to $N_{\text {eff }}$ is below the current limit from Planck, $\Delta N_{\text {eff }} \lesssim 0.3$ [10], provided $m_{N} \gtrsim 10 \mathrm{MeV}$ [11]. Our analysis still applies to this case if the lifetime of $N, \tau_{N} \sim 256 \pi^{3} \Lambda^{4} / m_{N}^{5}$ is larger than the scales relevant for the experiments considered in this work. Namely, for $m_{N} \lesssim 10^{-3} \Lambda^{4 / 5}$. Thus, for $\Lambda \gtrsim v, N$ behaves also as a stable particle if $m_{N} \lesssim 0.1 \mathrm{GeV}$. In summary, our study works within the $\nu$ SMEFT if the cutoff is above the EW scale and provided neutrinos are Dirac particles, or Majorana particles with $0.01 \mathrm{GeV} \lesssim m_{N} \lesssim 0.1 \mathrm{GeV}$; the upper limit being significantly larger if the EFT starts being valid only above the TeV.

This article is organised as follows. In section 2 we introduce the formalism of the $\nu$ SMEFT. We discuss which operators contribute to each of the observables considered 
in this work and compute the relevant equations. In section 3 we compare the obtained results with the experiments, and set global constraints on the (four-fermion) operators of the $\nu$ SMEFT. We emphasise that some of them are actually not bounded by current data, and thus develop a new collider search designed for the HL-LHC in section 4. We conclude in section 5 .

\section{Formalism}

The basis of dimension-six operators containing $N$ and respecting lepton and baryon numbers is shown in table 1. In the following subsections, we compute different cross sections and decay widths involving $N$ in the final state. Therefore, up to corrections proportional to the neutrino masses, the $\nu \mathrm{SMEFT}$ operators mediating the corresponding processes do not interfere with their SMEFT counterparts. As a result, any bounds computed on the $\nu$ SMEFT Wilson coefficients by using solely our equations are conservative. Hereafter, we assume all these coefficients to be real. For simplicity, we also restrict to only one $N$ family; expressions for the case of several $N$ fields can be trivially obtained from our results. Moreover, we explicitly do not consider the operators involving flavour violation between the first and second fermion families.

We focus mostly on observables sensitive to the four-fermion operators in the classes RRRR, LLRR and LRRL, where L (R) denotes left (right) handed fermions. These are typically more relevant because they can be generated at tree level in UV completions of the SM. Moreover, operators such as $\mathcal{O}_{N B}$ and $\mathcal{O}_{N W}$ are very much constrained by measurements of neutrino dipole moments when the latter are Dirac $[12,13]$. In the Majorana case they must be suppressed in order for $N$ to be long-lived; otherwise it would decay rather promptly into two body final states $[14,15]$. In such case, the collider signals are very different from the ones considered in this article and related works [16-18]; they will be presented elsewhere. We only include the contribution of the SF operators (see table 1) in the relevant equations hereafter for the sake of completeness.

For the observables computed using collider simulations we rely on MadGraph v5 [19], Pythia v8 [20, 21] and FastJet v3 [22], with a model containing the interactions in table 1 implemented using FeynRules v2 [23]. (For the operator $\mathcal{O}_{l d q N}$, we use the Fierztransformed version $\mathcal{O}_{l d q N}=1 / 2\left(\overline{q_{L}} d_{R}\right) \epsilon\left(\overline{l_{L}} N\right)+1 / 8\left(\overline{q_{L}} \sigma_{\mu \nu} d_{R}\right) \epsilon\left(\overline{l_{L}} \sigma^{\mu \nu} N\right)$.)

\subsection{Searches for one lepton and missing energy at the LHC}

When restricted to the first (and to a lesser extent, the second) quark family, the operators $\mathcal{O}_{d u N e}, \mathcal{O}_{l d q N}, \mathcal{O}_{l N q d}, \mathcal{O}_{q u N l}, \mathcal{O}_{H N e}$ and $\mathcal{O}_{N W}$ contribute to the production of $\ell+\mathbb{E}_{T}$ from quarks. In particular,

$$
\begin{aligned}
\frac{d \sigma}{d t}\left(u \bar{d} \rightarrow \ell_{i}^{+} N\right)=\frac{1}{192 \pi \Lambda^{4} s^{2}}\left\{\left[\left(\alpha_{q u N l}^{11 i}\right)^{2}+4\left(\alpha_{d u N e}^{11 i}\right)^{2}+\left(\alpha_{l N q d}^{i 11}\right)^{2}\right] s^{2}\right. & \\
+ & {\left[4\left(\alpha_{d u N e}^{11 i}\right)^{2}+\left(\alpha_{l d q N}^{i 11}\right)^{2}\right] t^{2}+2\left[4\left(\alpha_{d u N e}^{11 i}\right)^{2}-\alpha_{l N q d}^{i 11} \alpha_{l d q N}^{i 11}\right] s t } \\
+ & \left.4\left(\alpha_{H N e}^{i}\right)^{2} m_{W}^{4} \frac{t^{2}}{s^{2}}-32\left(\alpha_{N W}^{i}\right)^{2} m_{W}^{2}\left(\frac{t^{2}}{s}+t\right)\right\}
\end{aligned}
$$




\begin{tabular}{|c|ccc|}
\hline & $400-600$ & $600-1000$ & $1000-2000[\mathrm{GeV}]$ \\
\hline $\mathcal{A}_{1}$ & $7400(4400)$ & $12000(7200)$ & $16000(9600)$ \\
$\mathcal{A}_{2}$ & $2100(1300)$ & $3600(2200)$ & $4700(2800)$ \\
$\mathcal{A}_{3}$ & $-3500(-2100)$ & $-5600(-3400)$ & $-7700(-4600)$ \\
$\mathcal{A}_{4}$ & $5.0(3.0)$ & $1.2(0.73)$ & $0.15(0.089)$ \\
$\mathcal{A}_{5}$ & $360(210)$ & $210(120)$ & $79(47)$ \\
\hline $\mathrm{SM}$ & $9700 \pm 500(6460 \pm 330)$ & $2010 \pm 140(1320 \pm 90)$ & $232 \pm 24(150 \pm 13)$ \\
data & $9551(6772)$ & $1931(1392)$ & $246(177)$ \\
$s_{\max }$ & $791(778)$ & $213(257)$ & $67(62)$ \\
\hline
\end{tabular}

Table 2. Coefficients in $\mathrm{TeV}^{4}$ and rounded to two significant figures for $p p \rightarrow \ell N$ obtained upon recasting the experimental analysis of ref. [24] for $\mathcal{L}=36 \mathrm{fb}^{-1}$. The numbers outside (inside) the parentheses refer to the $\ell=e(\mu)$ case.

where $i=1$ (2) for electron (muon). The imprint of these operators in searches for $\ell+\mathbb{E}_{T}$ can be better observed in the tail of the distribution of the transverse mass of the lepton because, contrary to the SM background, the cross section of the signal grows with the energy due to the absence of propagator suppression.

A number of searches have been carried out at the LHC in this regard. Here, we focus on the ATLAS study of ref. [24], based on $36 \mathrm{fb}^{-1}$ of data collected at $\sqrt{s}=13 \mathrm{TeV}$. The main selection criteria are the requirement of exactly one light lepton with $p_{T}>55 \mathrm{GeV}$ (65 GeV) in the muon (electron) channel; and likewise for $\mathbb{E}_{T}$. The events are subsequently categorised according to the variable $m_{T}$, with $m_{T}^{2}=2 p_{T} \mathbb{E}_{T}\left(1-\cos \phi_{\ell \mathbb{E}_{T}}\right)$.

The numbers of predicted SM events and of observed events in four $m_{T}$ regions as provided in ref. [24] are shown in table 2. For convenience, we also show the maximum number of signal events, $s_{\max }$, in each bin separately. We have obtained them using the $\mathrm{CL}_{s}$ method [25] including the quoted uncertainties.

Following eq. 2.1, the number of events in the signal side in any of the bins can be expressed as:

$$
\begin{gathered}
N=\frac{1}{\Lambda^{4}}\left\{\left[\left(\alpha_{q u N l}^{11 i}\right)^{2}+4\left(\alpha_{d u N e}^{11 i}\right)^{2}+\left(\alpha_{l N q d}^{i 11}\right)^{2}\right] \mathcal{A}_{1}+\left[4\left(\alpha_{d u N e}^{11 i}\right)^{2}+\left(\alpha_{l d q N}^{i 11}\right)^{2}\right] \mathcal{A}_{2}\right. \\
\left.+2\left[4\left(\alpha_{d u N e}^{11 i}\right)^{2}-\alpha_{l N q d}^{i 11} \alpha_{l d q N}^{i 11}\right] \mathcal{A}_{3}+\left(\alpha_{H N e}^{i}\right)^{2} \mathcal{A}_{4}+\left(\alpha_{N W}^{i}\right)^{2} \mathcal{A}_{5}\right\}
\end{gathered}
$$

where $\mathcal{A}_{1}, \mathcal{A}_{2}, \cdots, \mathcal{A}_{5}$ are bin-dependent coefficients to be determined by simulation after recasting the experimental analysis. Our findings are also reported in table 2 . The values of the $\mathcal{A}$ coefficients for electrons and muons differ only by a factor of 0.6 , introduced to simulate the smaller muon detection efficiency due to the strict trigger and muon selection criteria of the experimental analysis. For the same operators with taus instead of light leptons, we recast the CMS analysis of ref. [26], based on $35.9 \mathrm{fb}^{-1}$ of data collected at $\sqrt{s}=13 \mathrm{TeV}$. More importantly, it requires a hadronic tau with $p_{T}>80 \mathrm{GeV}$, as well as $\mathbb{E}_{T}>200 \mathrm{GeV}$. This analysis divides the transverse mass range into slightly different bins; see table 3 which, as in the previous case, also reports the number of predicted SM events, the observed number of events and $s_{\max }$, as well as the values of the different coefficients (this time dubbed $\mathcal{B}$ ) as obtained from simulation. 


\begin{tabular}{|c|ccc|}
\hline & $0-500$ & $500-1000$ & $>1000[\mathrm{GeV}]$ \\
\hline $\mathcal{B}_{1}$ & 170 & 3600 & 10000 \\
$\mathcal{B}_{2}$ & 40 & 990 & 3200 \\
$\mathcal{B}_{3}$ & -69 & -1600 & -4700 \\
$\mathcal{B}_{4}$ & 0.33 & 1.0 & 0.15 \\
$\mathcal{B}_{5}$ & 40 & 290 & 160 \\
\hline $\mathrm{SM}$ & $1243 \pm 160$ & $485 \pm 77$ & $23.4 \pm 6.2$ \\
data & 1203 & 452 & 15 \\
$s_{\max }$ & 258 & 125 & 12 \\
\hline
\end{tabular}

Table 3. Coefficients in $\mathrm{TeV}^{4}$ and rounded to two significant figures for $p p \rightarrow \tau N$ obtained upon recasting the experimental analysis of ref. [26] for $\mathcal{L}=35.9 \mathrm{fb}^{-1}$. Note that eq. 2.1 is obtained under the assumption $\sqrt{s} \gg m_{W}$, so $\mathcal{B}_{4,5}$ in the first bin should not be taken rigorously.

In principle, the same operators but involving second generation quarks also contribute (without interfering with the previous ones) to this process. However, the corresponding $\mathcal{A}$ and $\mathcal{B}$ coefficients are expected to be a factor of $\sim 10$ smaller due to the smaller parton distribution functions (PDFs).

\subsection{Monojet searches at the LHC}

Four-fermion operators containing two light quarks and two RH neutrinos, i.e. $\mathcal{O}_{u N}, \mathcal{O}_{d N}$, $\mathcal{O}_{q N}$, can lead to monojet searches at the LHC if for example a gluon is emitted from one of the initial quarks in $p p$ collisions. They also modify the $\pi^{0}$ width but chirality suppressed. Furthermore, this width is experimentally known to be sensibly large [27].

Different monojet searches have been performed by ATLAS and CMS. In this work, we consider the CMS analysis of ref. [28], based on $35.9 \mathrm{fb}^{-1}$ of data collected at $\sqrt{s}=13 \mathrm{TeV}$. The main cuts of this study are missing transverse energy above $250 \mathrm{GeV}$, at least one hard jet with $p_{T}>250 \mathrm{GeV}$ and no isolated leptons. The analysis defines different signal regions depending on the value of $\mathbb{E}_{T}$; see table 4 .

The interference between the three aforementioned operators is chirality suppressed. Therefore, in very good approximation, one can estimate the number of signal events in any region of the analysis as

$$
N=\frac{1}{\Lambda^{4}}\left[\left(\alpha_{u N}^{11}\right)^{2} \mathcal{C}_{1}+\left(\alpha_{d N}^{11}\right)^{2} \mathcal{C}_{2}+\left(\alpha_{q N}^{11}\right)^{2} \mathcal{C}_{3}\right]
$$

with $\mathcal{C}_{1}, \mathcal{C}_{2}, \mathcal{C}_{3}$ depending again on the signal region.

Note that in this case we are neglecting the contribution of the operator $\mathcal{O}_{H N}$, which in principle interferes with the previous ones, because it does not grow at large energies. At any rate, as commented previously, we focus on scenarios with only four-fermion operators when setting bounds in section 3. Likewise, other four-fermion operators contribute to the monojet channel via $\nu N$. They of course do not interfere with the ones in eq. 2.3. Besides, they are constrained by other observables, so we conservatively neglect them.

\subsection{Pion decays}

The operators modifying the tail of $\ell+\mathbb{E}_{T}$ in $p p$ collisions can also enhance the pion decays, provided $N$ is the actual RH component of a Dirac neutrino. (Also if $N$ is Majorana with 


\begin{tabular}{|c|ccccc|}
\hline & $690-740$ & $740-790$ & $790-840$ & $840-900$ & $900-960[\mathrm{GeV}]$ \\
\hline $\mathcal{C}_{1}$ & 210 & 170 & 130 & 130 & 94 \\
$\mathcal{C}_{2}$ & 97 & 78 & 59 & 53 & 39 \\
$\mathcal{C}_{3}$ & 320 & 250 & 180 & 170 & 130 \\
\hline $\mathrm{SM}$ & $526 \pm 14$ & $325 \pm 12$ & $223 \pm 9$ & $169 \pm 8$ & $107 \pm 6$ \\
data & 557 & 316 & 233 & 172 & 101 \\
$s_{\max }$ & 82 & 40 & 44 & 35 & 21 \\
\hline
\end{tabular}

Table 4. Coefficients in $\mathrm{TeV}^{4}$ and rounded to two significant figures for $p p \rightarrow N \bar{N} g(q)$ obtained upon recasting the experimental analysis of ref. [28] for $\mathcal{L}=35.9 \mathrm{fb}^{-1}$.

low enough mass.) For the computation of the corresponding matrix elements, we neglect $\mathcal{O}_{l d q N}^{i 11}$, whose tensor form factor is hard to estimate. We obtain

$$
\begin{gathered}
\mathcal{M}\left(\pi^{-} \rightarrow \ell_{i}^{-} \bar{N}\right)=\frac{1}{\Lambda^{2}} \overline{u\left(p_{\ell_{i}}\right)}\{ \\
\left\langle 0\left|V^{\mu}\right| \pi^{-}\right\rangle\left[\left(\alpha_{d u N e}^{11 i}+\alpha_{H N e}^{i}\right) \gamma_{\mu}+2 \sqrt{2} \frac{\alpha_{N W}^{i}}{m_{W}}\left(\gamma_{\mu} \not p-p_{\mu}\right)\right] \\
\left.+\left\langle 0|S| \pi^{-}\right\rangle\left(\alpha_{q u N l}^{11 i}-\alpha_{l N q d}^{i 11}\right)\right\} P_{R} v\left(p_{N}\right),
\end{gathered}
$$

where $p=p_{\ell_{i}}+p_{N}$. A similar expression holds for $\pi^{+} \rightarrow \ell_{i}^{+} N$.

Following ref. [29], we assume the expectation values of the quark currents to be determined by current algebra: $\left\langle 0\left|V^{\mu}\right| \pi^{ \pm}\right\rangle=f_{\pi} p^{\mu}$ and $\left\langle 0|S| \pi^{ \pm}\right\rangle=f_{\pi} m_{\pi}^{2} /\left(m_{u}+m_{d}\right)$ with $f_{\pi} \sim 131 \mathrm{MeV}$. Then, the corresponding decay width reads

$$
\begin{aligned}
\Gamma\left(\pi^{-} \rightarrow \ell_{i}^{-} \bar{N}\right)= & \frac{f_{\pi}^{2} m_{\pi}}{16 \pi \Lambda^{4}}\left(1-\frac{m_{\ell_{i}}^{2}}{m_{\pi}^{2}}\right)^{2} \\
& \times\left[m_{\ell_{i}}\left(\alpha_{d u N e}^{11 i}+\alpha_{H N e}^{i}\right)+\frac{m_{\pi}^{2}}{m_{u}+m_{d}}\left(\alpha_{q u N l}^{11 i}-\alpha_{l N q d}^{i 11}\right)\right]^{2} .
\end{aligned}
$$

The measured values of the pion decay widths into electrons and muons read $\Gamma(\pi \rightarrow$ $e+$ inv $)=(310 \pm 1) \times 10^{-23} \mathrm{GeV}$ and $\Gamma(\pi \rightarrow \mu+$ inv $)=(25279 \pm 5) \times 10^{-21} \mathrm{GeV}$, respectively [27]. We bound the coefficients entering this equation by requiring that the corresponding contribution is smaller than twice the experimental error.

\subsection{Tau decays}

The following operators give contributions to $\tau \rightarrow \ell+$ inv:

$$
\begin{aligned}
\mathcal{O}_{e N}^{i 3} & =\left(\overline{\ell_{i R}} \gamma_{\mu} \tau_{R}\right)\left(\bar{N} \gamma^{\mu} N\right), \\
\mathcal{O}_{l N}^{i 3} & =\left(\overline{\nu_{i L}} \gamma_{\mu} \nu_{\tau L}\right)\left(\bar{N} \gamma^{\mu} N\right)+\left(\overline{\ell_{\boldsymbol{i L}}} \gamma_{\boldsymbol{\mu}} \boldsymbol{\tau}_{\boldsymbol{L}}\right)\left(\overline{\boldsymbol{N}} \gamma^{\mu} \boldsymbol{N}\right), \\
\mathcal{O}_{l N l e}^{i i 3} & =\left(\overline{\boldsymbol{\nu}_{\boldsymbol{i}}} \boldsymbol{N}\right)\left(\overline{\ell_{\boldsymbol{i} \boldsymbol{L}}} \boldsymbol{\tau}_{\boldsymbol{R}}\right)-\left(\overline{\ell_{\boldsymbol{i}}} \boldsymbol{N}\right)\left(\overline{\boldsymbol{\nu}_{\boldsymbol{i} \boldsymbol{L}}} \boldsymbol{\tau}_{\boldsymbol{R}}\right)+\text { h.c. }, \\
\mathcal{O}_{l N l e}^{i 3 i} & =\left(\overline{\boldsymbol{\nu}_{\boldsymbol{i}}} \boldsymbol{N}\right)\left(\overline{\boldsymbol{\tau}_{\boldsymbol{L}}} \ell_{\boldsymbol{i} \boldsymbol{R}}\right)-\left(\overline{\ell_{i L}} N\right)\left(\overline{\nu_{\tau L}} \ell_{i R}\right)+\text { h.c. }, \\
\mathcal{O}_{l N l e}^{3 i i} & =\left(\overline{\nu_{\tau L}} N\right)\left(\overline{\ell_{i L}} \ell_{i R}\right)-\left(\overline{\boldsymbol{\tau}_{\boldsymbol{L}}} \boldsymbol{N}\right)\left(\overline{\boldsymbol{\nu}_{\boldsymbol{i}}} \ell_{\boldsymbol{i} \boldsymbol{R}}\right)+\text { h.c. },
\end{aligned}
$$




$$
\begin{aligned}
& \mathcal{O}_{l N l e}^{i 33}=\left(\overline{\nu_{i L}} N\right)\left(\overline{\tau_{L}} \tau_{R}\right)-\left(\overline{\ell_{\boldsymbol{i L}}} \boldsymbol{N}\right)\left(\overline{\boldsymbol{\nu}_{\boldsymbol{\tau} \boldsymbol{L}} \boldsymbol{\tau}_{\boldsymbol{R}}}\right)+\text { h.c. } \\
& \mathcal{O}_{l N l e}^{3 i 3}=\left(\overline{\boldsymbol{\nu}_{\boldsymbol{\tau} \boldsymbol{L}}} \boldsymbol{N}\right)\left(\overline{\ell_{\boldsymbol{i} \boldsymbol{L}}} \boldsymbol{\tau}_{\boldsymbol{R}}\right)-\left(\overline{\tau_{L}} N\right)\left(\overline{\nu_{i L}} \tau_{R}\right)+\text { h.c. } \\
& \mathcal{O}_{l N l e}^{33 i}=\left(\overline{\boldsymbol{\nu}_{\boldsymbol{\tau} \boldsymbol{L}}} \boldsymbol{N}\right)\left(\overline{\boldsymbol{\tau}_{\boldsymbol{L}}} \ell_{\boldsymbol{i} \boldsymbol{R}}\right)-\left(\overline{\boldsymbol{\tau}_{\boldsymbol{L}}} \boldsymbol{N}\right)\left(\overline{\boldsymbol{\nu}_{\boldsymbol{\tau} \boldsymbol{L}}} \ell_{\boldsymbol{i} \boldsymbol{R}}\right)+\text { h.c. } \\
& \mathcal{O}_{H N e}^{i} \supset \frac{v m_{W}}{\sqrt{2}}\left(\bar{N} \gamma_{\mu} \ell_{i R}\right) W^{+\mu}+\text { h.c. } \\
& \mathcal{O}_{H N e}^{3} \supset \frac{v m_{W}}{\sqrt{2}}\left(\bar{N} \gamma_{\mu} \tau_{R}\right) W^{+\mu}+\text { h.c. } \\
& \mathcal{O}_{N W}^{i} \supset 2 v\left(\overline{\ell_{i L}} \sigma_{\mu \nu} N\right) \partial^{\mu} W^{-\nu}+\text { h.c. } \\
& \mathcal{O}_{N W}^{3} \supset 2 v\left(\overline{\tau_{L}} \sigma_{\mu \nu} N\right) \partial^{\mu} W^{-\nu}+\text { h.c. }
\end{aligned}
$$

When there are more than one term, only those in boldface are relevant. These operators lead to the following decay widths of the tau lepton:

$$
\begin{aligned}
& \Gamma\left(\tau \rightarrow \ell_{i} \bar{N} N\right)=\frac{m_{\tau}^{5}}{1536 \pi^{3} \Lambda^{4}} {\left[\left(\alpha_{e N}^{i 3}\right)^{2}+\left(\alpha_{l N}^{i 3}\right)^{2}\right], } \\
& \Gamma\left(\tau \rightarrow \ell_{i} \bar{N} \nu_{i}\right)=\frac{m_{\tau}^{5}}{6144 \pi^{3} \Lambda^{4}}\left(\alpha_{l N l e}^{i i 3}\right)^{2} \\
& \Gamma\left(\tau \rightarrow \ell_{i} \overline{\nu_{i}} N\right)=\frac{m_{\tau}^{5}}{6144 \pi^{3} \Lambda^{4}}\left[\left(\alpha_{l N l e}^{i 3 i}\right)^{2}+\left(\alpha_{l N l e}^{3 i i}\right)^{2}-\alpha_{l N l e}^{i 3 i} \alpha_{l N l e}^{3 i i}+4\left(\alpha_{H N e}^{3}\right)^{2}\right. \\
&\left.+\frac{64}{5} \frac{m_{\tau}^{2}}{m_{W}^{2}}\left(\alpha_{N W}^{3}\right)^{2}+8 \sqrt{2} \frac{m_{\tau}}{m_{W}} \alpha_{H N e}^{3} \alpha_{N W}^{3}\right], \\
& \Gamma\left(\tau \rightarrow \ell_{i} \bar{N} \nu_{\tau}\right)=\frac{m_{\tau}^{5}}{6144 \pi^{3} \Lambda^{4}}\left[\left(\alpha_{l N l e}^{i 33}\right)^{2}+\left(\alpha_{l N l e}^{3 i 3}\right)^{2}-\alpha_{l N l e}^{i 33} \alpha_{l N l e}^{333}+4\left(\alpha_{H N e}^{i}\right)^{2}\right. \\
&\left.\quad+\frac{24}{5} \frac{m_{\tau}^{2}}{m_{W}^{2}}\left(\alpha_{N W}^{i}\right)^{2}-2 \sqrt{2} \frac{m_{\tau}}{m_{W}} \alpha_{l N l e}^{3 i 3} \alpha_{N W}^{i}\right], \\
& \Gamma\left(\tau \rightarrow \ell_{i} \overline{\nu_{\tau}} N\right)=\frac{m_{\tau}^{5}}{6144 \pi^{3} \Lambda^{4}}\left(\alpha_{l N l e}^{33 i}\right)^{2} .
\end{aligned}
$$

The measured values of the tau decay widths into electrons and muons are $\Gamma(\tau \rightarrow$ $e+$ inv $)=(4.03 \pm 0.02) \times 10^{-13} \mathrm{GeV}$ and $\Gamma(\tau \rightarrow \mu+$ inv $)=(3.93 \pm 0.02) \times 10^{-13} \mathrm{GeV}$, respectively [27]. Following the same procedure as in the pion case, we bound these operators by requiring that the corresponding theoretical decay width is not larger than twice the experimental error.

\subsection{Top decays}

Finally, the following four-fermion operators contribute to flavour-conserving top decays:

$$
\begin{aligned}
\mathcal{O}_{d u N e}^{33 i}= & \left(\overline{b_{R}} \gamma_{\mu} t_{R}\right)\left(\bar{N} \gamma^{\mu} \ell_{i R}\right)+\text { h.c. } \\
\mathcal{O}_{l d q N}^{i 33}= & \frac{\mathbf{1}}{\mathbf{2}}\left(\overline{\boldsymbol{t}_{\boldsymbol{L}}} \boldsymbol{b}_{\boldsymbol{R}}\right)\left(\overline{\boldsymbol{\ell}_{\boldsymbol{i L}}} \boldsymbol{N}\right)-\frac{1}{2}\left(\overline{b_{L}} b_{R}\right)\left(\overline{\nu_{i L}} N\right) \\
& +\frac{\mathbf{1}}{\mathbf{8}}\left(\overline{\boldsymbol{t}_{\boldsymbol{L}}} \boldsymbol{\sigma}_{\boldsymbol{\mu} \boldsymbol{\nu}} \boldsymbol{b}_{\boldsymbol{R}}\right)\left(\overline{\boldsymbol{\ell}_{\boldsymbol{i}}} \boldsymbol{\sigma}^{\boldsymbol{\mu} \boldsymbol{\nu}} \boldsymbol{N}\right)-\frac{1}{8}\left(\overline{b_{L}} \sigma_{\mu \nu} b_{R}\right)\left(\overline{\nu_{i L}} \sigma^{\mu \nu} N\right)+\text { h.c. }
\end{aligned}
$$




$$
\begin{aligned}
\mathcal{O}_{l N q d}^{i 33} & =\left(\overline{\nu_{i L}} N\right)\left(\overline{b_{L}} b_{R}\right)-\left(\overline{\boldsymbol{\ell}_{\boldsymbol{i} \boldsymbol{L}}} \boldsymbol{N}\right)\left(\overline{\boldsymbol{t}_{\boldsymbol{L}}} \boldsymbol{b}_{\boldsymbol{R}}\right)+\text { h.c. } \\
\mathcal{O}_{q u N l}^{33 i} & =\left(\overline{t_{L}} t_{R}\right)\left(\bar{N} \nu_{i L}\right)+\left(\overline{\boldsymbol{b}_{\boldsymbol{L}}} \boldsymbol{t}_{\boldsymbol{R}}\right)\left(\overline{\boldsymbol{N}} \boldsymbol{\ell}_{\boldsymbol{i} \boldsymbol{L}}\right)+\text { h.c. }
\end{aligned}
$$

When there are more than one term, only those in boldface are relevant. The corresponding decay width reads

$$
\begin{aligned}
\Gamma\left(t \rightarrow b \ell_{i}^{+} N\right)=\frac{m_{t}^{5}}{6144 \pi^{3} \Lambda^{4}}\left[4\left(\alpha_{d u N e}^{33 i}\right)^{2}+\left(\alpha_{q u N l}^{33 i}\right)^{2}\right. & \\
& \left.+\left(\alpha_{l d q N}^{i 33}\right)^{2}+\left(\alpha_{l N q d}^{i 33}\right)^{2}-\alpha_{l d q N}^{i 33} \alpha_{l N q d}^{i 33}\right]
\end{aligned}
$$

and similarly for $\bar{t} \rightarrow \bar{b} \ell_{i}^{-} \bar{N}$. In this expression, we have neglected the contribution of $\mathcal{O}_{H N e}$ and $\mathcal{O}_{N W}$. The reason is that in those cases the top decay is approximately two body, followed by the leptonic decay of an on-shell $W$. This does not only allow to disentangle the two contributions, but the interference is also very small. Similar results have been pointed out in the SMEFT; see e.g. ref. [30].

These operators can be mostly probed only in top decays. In particular, $\mathcal{O}_{d u N e}$ does not provide any other interaction. This is in contrast with analogous operators in the SMEFT, such as for example $\sim\left(\overline{t_{R}} \gamma_{\mu} t_{R}\right)\left(\overline{l_{L}} \gamma^{\mu} l_{L}\right)$, because the left-handed (LH) neutrinos always come along with charged leptons. These operators are therefore better tested at lepton facilities; see e.g. ref. [31].

This discussion applies also to the flavour-violating top operators:

$$
\begin{aligned}
\mathcal{O}_{u N}^{13} & =\left(\overline{\boldsymbol{u}_{\boldsymbol{R}}} \gamma_{\boldsymbol{\mu}} \boldsymbol{t}_{\boldsymbol{R}}\right)\left(\overline{\boldsymbol{N}} \gamma^{\boldsymbol{\mu}} \boldsymbol{N}\right) \\
\mathcal{O}_{q N}^{13} & =\left(\overline{\boldsymbol{u}_{\boldsymbol{L}}} \gamma_{\boldsymbol{\mu}} \boldsymbol{t}_{\boldsymbol{L}}\right)\left(\overline{\boldsymbol{N}} \gamma^{\boldsymbol{\mu}} \boldsymbol{N}\right)+\left(\overline{d_{L}} \gamma_{\mu} b_{L}\right)\left(\bar{N} \gamma^{\mu} N\right), \\
\mathcal{O}_{q u N l}^{13 i} & =\left(\overline{\boldsymbol{u}_{\boldsymbol{L}}} \boldsymbol{t}_{\boldsymbol{R}}\right)\left(\overline{\boldsymbol{N}} \boldsymbol{\nu}_{\boldsymbol{i} \boldsymbol{L}}\right)+\left(\overline{d_{L}} t_{R}\right)\left(\bar{N} \ell_{i L}\right)+\text { h.c. } \\
\mathcal{O}_{q u N l}^{31 i} & =\left(\overline{\boldsymbol{t}_{\boldsymbol{L}}} \boldsymbol{u}_{\boldsymbol{R}}\right)\left(\overline{\boldsymbol{N}} \boldsymbol{\nu}_{\boldsymbol{i} \boldsymbol{L}}\right)+\left(\overline{b_{L}} u_{R}\right)\left(\bar{N} \ell_{i L}\right)+\text { h.c. }
\end{aligned}
$$

The terms in boldface lead to the rare top decays $t \rightarrow j+$ inv (where $j$ stands for $j e t$ ); again without charged lepton counterpart unlike the SMEFT [32, 33]. The corresponding decay widths read:

$$
\begin{aligned}
\Gamma(t \rightarrow u \bar{N} N) & =\frac{m_{t}^{5}}{1536 \pi^{3} \Lambda^{4}}\left[\left(\alpha_{u N}^{13}\right)^{2}+\left(\alpha_{q N}^{13}\right)^{2}\right] \\
\Gamma\left(t \rightarrow u \overline{\nu_{i}} N\right) & =\frac{m_{t}^{5}}{6144 \pi^{3} \Lambda^{4}}\left(\alpha_{q u N l}^{13 i}\right)^{2} \\
\Gamma\left(t \rightarrow u \bar{N} \nu_{i}\right) & =\frac{m_{t}^{5}}{6144 \pi^{3} \Lambda^{4}}\left(\alpha_{q u N l}^{31 i}\right)^{2} .
\end{aligned}
$$

Similar expressions hold of course for second generation quarks. The sensitivity of measurements at colliders is expected to be similar in both cases, just as in other flavourviolating top decays [33-38].

Current bounds on the top width are not constraining enough in any of these cases, though. Instead, dedicated analyses at colliders are to be performed to bound these operators. In section 4 we develop one such analysis for the flavour-conserving top decay at 
the LHC. The flavour-violating processes involve a light quark and two sources of missing energy, making these much appropriate search channels at future lepton colliders, in which all the three components of the missing momentum are measured.

\section{Global constraints}

Taking into account the observables computed in eqs. 2.2 (for light leptons and taus; see tables 2 and 3 , respectively), 2.3, 2.5 and 2.18-2.22, we can set constraints on the different operators of table 1 . The bounds are given by the non-boldfaced numbers in tables $5-7$.

When relying on high energy searches at colliders, we derive the bounds on the operator coefficients from the several bins for each of the analyses and keep the bounds yielding the strongest result. However, we also ensure that we do not go very high in energies in order to abide by EFT validities. Specifically, we remain within energy bins of less than $1 \mathrm{TeV}$.

Deriving the bounds on $\alpha_{u N}^{11}, \alpha_{d N}^{11}$ and $\alpha_{q N}^{11}$ is particularly simple as they enter the analytical expression for the number of events, for the monojet analysis, without any interference. In this case, the $\mathbb{E}_{T}$ bin of [740-790] GeV yields the strongest limits.

For the $\ell+\mathbb{E}_{T}$ analysis, it is straightforward to constrain $\alpha_{q u N l}^{11 i}$ and $\alpha_{d u N e}^{11 i}$ as they do not interfere with any operator in the expression for the number of events. For the operators that do interfere, viz. $\mathcal{O}_{l N q d}^{i 11}$ and $\mathcal{O}_{l d q N}^{i 11}$, we marginalise over each coefficient to set a bound on the second one. Thus, in order to constrain $\alpha_{l d q N}^{i 11}$, we fix $\alpha_{l N q d}^{i 11}$ to the value that minimises the number of events for each value of $\alpha_{l d q N}^{i 11}$, from where the procedure of setting bounds reduces to the one dimension as in the previous cases. The reverse process can be followed to constrain $\alpha_{l N q d}^{i 11}$.

The bounds obtained this way must be taken if $N$ is a Majorana neutrino with mass above $m_{\pi}$. For Dirac neutrinos, we bound $\alpha_{l N q d}^{i 11}$ instead by using the pion decay width in eq. 2.5 (neglecting the $m_{\ell_{i}}$ piece). The corresponding limit is a factor of 1.9 (1.8) more stringent for electrons (muons) than the LHC counterpart. This is the constraint we show in tables. Operators involving electrons are more constrained than those involving muons due to the larger sensitivity of the analysis of ref. [24] to electrons. Also, the pion decay width to $\mu+$ inv is significantly larger than that for $e+$ inv, therefore leaving more space for new physics.

The $\tau+\mathbb{E}_{T}$ follows a similar technique and helps us bound $\alpha_{q u N l}^{113}, \alpha_{d u N e}^{113}, \alpha_{l d q N}^{311}$ and $\alpha_{l N q d}^{311}$. We use the respective bins of [600-1000] GeV in $m_{T}$ for the $\ell+\mathbb{E}_{T}$ analysis and [500-1000] GeV for the $\tau+\mathbb{E}_{T}$ case. When the aforementioned operators are considered with second generation quarks instead of the first family, the bounds get weaker by a factor of $\sim 3$. Thus, without relying on flavour observables, one can estimate the bounds on operators involving transitions between the first and second quark families to be those quoted in the tables up to a factor of $\sim 1-3$. (Flavour observables can be much more constraining, though.)

Finally, we bound operators modifying the tau decays into electrons and muons. $\mathcal{O}_{e N}^{i 3}$, $\mathcal{O}_{l N}^{i 3}, \mathcal{O}_{l N l e}^{i i 3}$ and $\mathcal{O}_{l N l e}^{33 i}$ can be straightforwardly constrained, because they do not interfere with any other operator. $\mathcal{O}_{\text {lNle }}^{i 3 i}$ and $\mathcal{O}_{\text {lNle }}^{3 i i}$ interfere among themselves; we bound each by marginalising over the other. Likewise for $\mathcal{O}_{l N l e}^{i 33}$ and $\mathcal{O}_{l N l e}^{3 i 3}$. In this case, muonic and 


\begin{tabular}{|l|ccc|}
\hline Operator & $\alpha_{\max }$ for $\Lambda=1 \mathrm{TeV}$ & $\Lambda_{\min }[\mathrm{TeV}]$ for $\alpha=1$ & Observable \\
\hline $\mathcal{O}_{e N}^{i 3}$ & $3.0(2.9)$ & $0.58(0.59)$ & $\tau \rightarrow \ell+$ inv \\
\hline $\mathcal{O}_{d N}^{11}$ & 0.72 & 1.2 & monojet \\
\hline $\mathcal{O}_{u}^{11}$ & 0.48 & 1.4 & monojet \\
\hline $\mathcal{O}_{u N}^{11}$ & $0.11(0.16)$ & $3.0(2.5)$ & $\ell+\mathbb{E}_{T}^{-}$ \\
$\mathcal{O}_{d u N e}^{11 i}$ & 0.15 & 2.6 & $\tau+\mathbb{E}_{T}$ \\
$\mathcal{O}_{d u N e}^{113}$ & $\mathbf{9 . 2}(\mathbf{9 . 2}$ & $\mathbf{0 . 3 3}(\mathbf{0 . 3 3})$ & $\boldsymbol{t} \rightarrow \boldsymbol{b} \boldsymbol{\ell}+\mathbf{i n v}$ \\
$\mathcal{O}_{d u N e}^{33 i}$ & &
\end{tabular}

Table 5. Maximum (minimum) value of $\alpha(\Lambda)$ for $\Lambda=1 \mathrm{TeV}(\alpha=1)$ allowed by the observables quoted in the last column for $R R R R$ operators. The numbers outside (inside) the parentheses refer to $\ell=e(\mu)$.

\begin{tabular}{|l|ccc|}
\hline Operator & $\alpha_{\max }$ for $\Lambda=1 \mathrm{TeV}$ & $\Lambda_{\min }[\mathrm{TeV}]$ for $\alpha=1$ & Observable \\
\hline $\mathcal{O}_{l N}^{i 3}$ & $3.0(2.9)$ & $0.58(0.59)$ & $\tau \rightarrow \ell+$ inv \\
\hline $\mathcal{O}_{q N}^{11}-$ & 0.40 & 1.6 & monojet \\
\hline
\end{tabular}

Table 6. Maximum (minimum) value of $\alpha(\Lambda)$ for $\Lambda=1 \mathrm{TeV}(\alpha=1)$ allowed by the observables quoted in the last column for $L L R R$ operators. The numbers outside (inside) the parentheses refer to $\ell=e(\mu)$.

electronic operators get more or less equally constrained. Similar operators but involving transitions between the first and second lepton families could be constrained using muon decays. Naively, the bounds would be a factor of 10-100 stronger. However, the Fermi constant would be also redefined on account of the Wilson coefficients, making the process of bounding these operators more subtle.

Among those operators not constrained by the observables considered in this paper, we find $\mathcal{O}_{N N}$, which only involves $\mathrm{RH}$ neutrinos. We also have $\mathcal{O}_{e N}^{i i}$ and $\mathcal{O}_{l N}^{i i}$; these could be tested in monophoton searches at lepton colliders. Third generation flavour conserving $\mathcal{O}_{d N}^{33}, \mathcal{O}_{u N}^{33}$ and $\mathcal{O}_{q N}^{33}$ could be tested in searches for $b \bar{b}+\mathbb{E}_{T}$ and $t \bar{t}+\mathbb{E}_{T}$. Most of the rest of operators induce new top decays, either into $b \ell+$ inv, $b \tau+$ inv or $j+$ inv. The latter one is hard to probe at hadron colliders, because it involves two sources of missing energy and only light jets. (Moreover, is flavour-violating.) The second one involves a tau lepton, making this signal less promising than the first one. The first signal gives rise to a final state which is identical to the SM leptonic top decay (which complicates its study), but interestingly in this case the lepton and the missing energy do not reconstruct a $W$ boson. This provides a completely new signal not yet explored experimentally, for which we design a completely novel search strategy. For completeness, we advance the prospective bounds on the corresponding operators by the boldfaced numbers in tables 5 and 7 .

\section{$4 \quad t \rightarrow b \ell N$ at the $\mathrm{LHC}$}

We can constrain the operators $\mathcal{O}_{d u N e}, \mathcal{O}_{\ell d q N}, \mathcal{O}_{\ell N q d}$ and $\mathcal{O}_{q u \ell N}$ in $t \bar{t}$ production with one of the tops decaying exactly as in the SM in the hadronic mode and the other decaying leptonically through the modified vertex. We focus on the high luminosity run of the LHC 


\begin{tabular}{|c|c|c|c|}
\hline Operator & $\alpha_{\max }$ for $\Lambda=1 \mathrm{TeV}$ & $\Lambda_{\min }[\mathrm{TeV}]$ for $\alpha=1$ & Observable \\
\hline $\mathcal{O}_{l N l e}^{i i 3}$ & $6.0(5.9)$ & $0.41(0.41)$ & $\tau \rightarrow \ell+\operatorname{inv}$ \\
\hline $\mathcal{O}_{l N l e}^{i 3 i}$ & $6.8(6.8)$ & $0.38(0.38)$ & $\tau \rightarrow \ell+$ inv \\
\hline $\mathcal{O}_{l N l e}^{i 33}$ & $6.8(6.8)$ & $0.38(0.38)$ & $\tau \rightarrow \ell+\operatorname{inv}$ \\
\hline $\mathcal{O}_{l N l e}^{3 i i}$ & $6.8(6.8)$ & $0.38(0.38)$ & $\tau \rightarrow \ell+\operatorname{inv}$ \\
\hline $\mathcal{O}_{l N l e}^{3 i 3}$ & $6.8(6.8)$ & $0.38(0.38)$ & $\tau \rightarrow \ell+\operatorname{inv}$ \\
\hline $\mathcal{O}_{l N l e}^{33 i}$ & $6.0(5.9)$ & $0.41(0.41)$ & $\tau \rightarrow \ell+\operatorname{inv}$ \\
\hline $\mathcal{O}_{l d q N}^{i 11}$ & $0.46(0.66)$ & $1.5(1.2)$ & $\ell+\mathbb{E}_{T}$ \\
\hline $\mathcal{O}_{l d q N}^{i 33}$ & $21(21)$ & $0.22(0.22)$ & $t \rightarrow b \ell+\mathrm{inv}$ \\
\hline $\mathcal{O}_{-}^{l d q} \underline{l d}_{-}$ & 0.67 & 1.2 & $\tau+\mathbb{E}_{T}$ \\
\hline $\mathcal{O}_{l N q d}^{i 11}$ & $0.25(0.36)$ & $2.0(1.7)$ & $\pi \rightarrow \ell+\operatorname{inv}$ \\
\hline $\mathcal{O}_{l N q d}^{i 33}$ & $21(21)$ & $0.22(0.22)$ & $t \rightarrow b \ell+\mathrm{inv}$ \\
\hline $\mathcal{O}_{-l N \underline{d} d}^{311}$ & 0.35 & 1.7 & $\tau+\mathbb{E}_{T}$ \\
\hline $\mathcal{O}_{q u N l}^{11 i}$ & $0.13(0.19)$ & $2.8(2.3)$ & $\ell+\mathbb{E}_{T}$ \\
\hline $\mathcal{O}_{q u N l}^{113}$ & 0.19 & 2.3 & $\tau+\mathbb{E}_{T}$ \\
\hline $\mathcal{O}_{q u N l}^{33 i}$ & $18(18)$ & $0.23(0.23)$ & $t \rightarrow b \ell+$ inv \\
\hline
\end{tabular}

Table 7. Maximum (minimum) value of $\alpha(\Lambda)$ for $\Lambda=1 \mathrm{TeV}(\alpha=1)$ allowed by the observables quoted in the last column for $L R R L$ operators. The numbers outside (inside) the parentheses refer to $\ell=e(\mu)$.

at $14 \mathrm{TeV}$ with an integrated luminosity of $3 \mathrm{ab}^{-1}$. The final state consists of $b \bar{b} \ell \mathbb{E}_{T}+$ jets. (We perform our analysis only in the muonic final state. We assume the results not to be significantly different for the case of electrons.) The dominant background to this process comes from the SM semi-leptonic $t \bar{t}$ channel. It is a very challenging task to reduce the background, as the topology is essentially the same in both the signal and the background. In particular, the hadronic top decays in the exact same manner in both cases.

Our analysis strategy starts by generating the signal and background events at $\sqrt{s}=$ $14 \mathrm{TeV}$ using the NNPDF23LO PDF set [39]. We generate our samples at the leading order and then multiply by a flat next-to-next-to leading order (NNLO) + next-to-next-to leading logarithm (NNLL) $k$-factor of $\sim 1.63$ corresponding to $m_{t}=172.5 \mathrm{GeV}$ and for the central value of the cross section. The central value of the $14 \mathrm{TeV}$ NNLO+NNLL cross section is $984.50 \mathrm{pb}[40]$. At the generation level, we demand $p_{T}(j, b, \ell)>15,15,10 \mathrm{GeV}$, $|\eta(j, b, \ell)|<5,3,3$ and $\Delta R(j j, b b, b j, j \ell, b \ell)>0.3$ and $\Delta R(\ell \ell)>0.2$. We shower the events using Pythia v8 [20, 21]. We construct jets upon using the anti-k $\mathrm{k}_{t}$ algorithm [41] with a jet parameter $R=0.4$ in the FastJet v3 framework [22]. We require the jets to have $p_{T}>30 \mathrm{GeV}$ and the $b$-tagged jets to have $|\eta|<2.5$. The leptons are required to have $p_{T}>10 \mathrm{GeV}$ and $|\eta|<2.5$. The $b$-tagged jets are constructed by requiring the $B$-meson tracks to be within $\Delta R=0.2$ of a jet. Moreover, we implement a flat $b$-tagging efficiency of $70 \%$. In our simplified analysis, we also consider $c$ quarks and light quarks faking a $b$-jet with probabilities of $10 \%$ and $1 \%$, respectively. Finally, isolated leptons are defined by requiring that the hadronic activity around $\Delta R=0.2$ of the corresponding lepton is smaller than $10 \%$ of its $p_{T}$. 

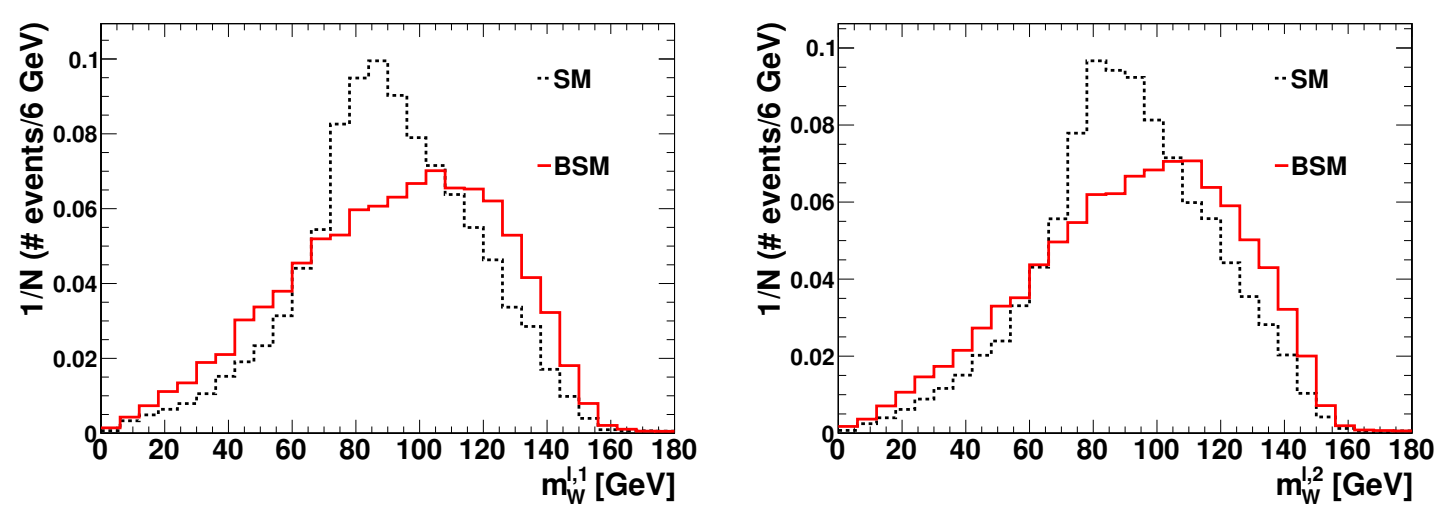

Figure 1. Reconstructed $W$-boson mass for the signal (solid red) and background (dashed black). Here 1 (2) refers to the "+" ("-") solution of the neutrino $p_{z}$ obtained while solving for the invariant mass of the leptonic top.

Subsequently, we demand exactly two $b$-tagged jets, one isolated lepton and at least two light jets. In order to ascertain proper top-mass reconstruction, we build two hadronic top mass variables $\left(m_{\text {had,1 }}^{t}\right.$ and $\left.m_{\text {had,2 }}^{t}\right)$. We include the two hardest light jets $\left(j_{1,2}\right)$ when reconstructing both $m_{\text {had,1 }}^{t}$ and $m_{\text {had,2. }}^{t}$. However, we include the harder (softer) $b$-tagged jet to reconstruct $m_{\text {had, } 1}^{t}\left(m_{\text {had }, 2}^{t}\right)$. For each event we check which of these reconstructed hadronic top masses is closest to the actual top mass, that we take to be $m_{t}=172.5 \mathrm{GeV}$. The $b$-jet giving the poorer reconstruction is assigned to the leptonic top. Next, we demand that the best reconstructed hadronic top $\left(m_{t}^{\text {had }}\right)$ and the hadronic $W\left(m_{W}^{\text {had }}\right)$, reconstructed out of the two hardest light jets, lie respectively within $40 \mathrm{GeV}$ and $30 \mathrm{GeV}$ of the top and $W$ masses (with $m_{W}$ taken as $80.385 \mathrm{GeV}$ ).

We reconstruct the $p_{z}$ of the neutrino by solving for the leptonic top mass. There is a two-fold ambiguity in this process, which results into two values for the momentum of the $\ell \nu$ system, corresponding to the "+" and "-" solutions of the quadratic equation. We denote these two solutions by $W_{1}$ and $W_{2}$, respectively. (Note, however, that in the signal they do not need to be close to the $W$ mass.) We only select those events where the solution to the quadratic equation for the top mass has a positive discriminant. The aforementioned trigger cuts, isolation cuts and analysis cuts have the respective efficiencies of $4.4 \%$ and $4.7 \%$ for the signal and the SM background.

The distribution of $m_{W}^{\ell, 1 / 2}$, where 1 (2) refers to the "+" ("-") solution of the aforementioned quadratic equation, is shown in figure 1 . Despite the difference in $m_{W}^{\ell, 1 / 2}$ (that relies on the fact that there is no $W$ for the leptonic top in the EFT scenario), the signal and the background distributions are apparently not very different from the point of view of a cut-and-count analysis. We therefore perform a simple boosted decision tree (BDT) analysis in the TMVA framework [42]. We include the following observables: the four-momenta of the lepton and the $b$-tagged jet best reconstructing the leptonic top, the two components of the transverse missing momentum, $m_{W}^{\text {had }}, m_{W}^{\ell, 1}, m_{W}^{\ell, 2}, m_{t}^{\text {had }}, \mathbb{E}_{T}, \Delta R_{b, b}, \Delta R_{b_{\ell}, j_{1 / 2}}, \Delta R_{\ell, b_{h}}$ and $\Delta R_{\ell, j_{1 / 2}}$, where $b_{\ell}\left(b_{h}\right)$ is the $b$-jet associated with the leptonic (hadronic) top. We ensure that there is no overtraining by checking that the Kolmogorov-Smirnov statistic for neither the signal nor the background falls below the critical value of 0.01 [43]. As 


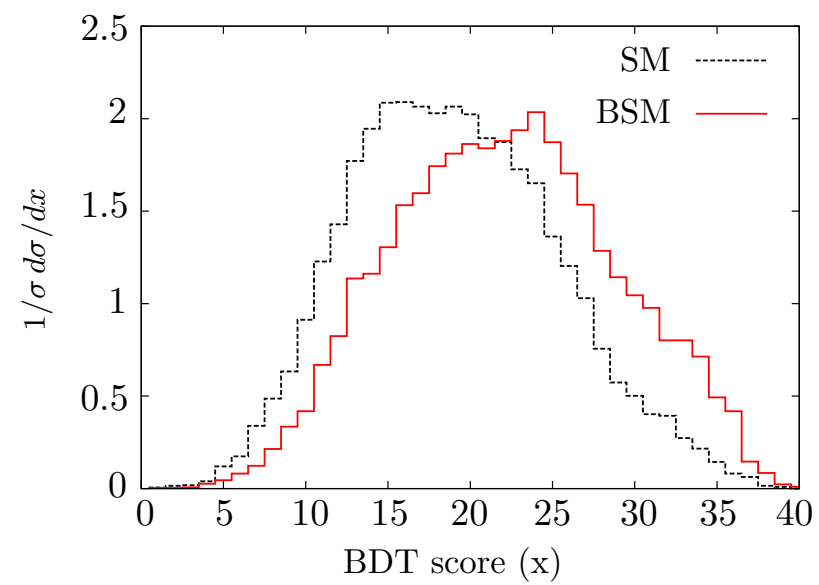

Figure 2. BDT score variable used in the asymmetry determination as described in the text.
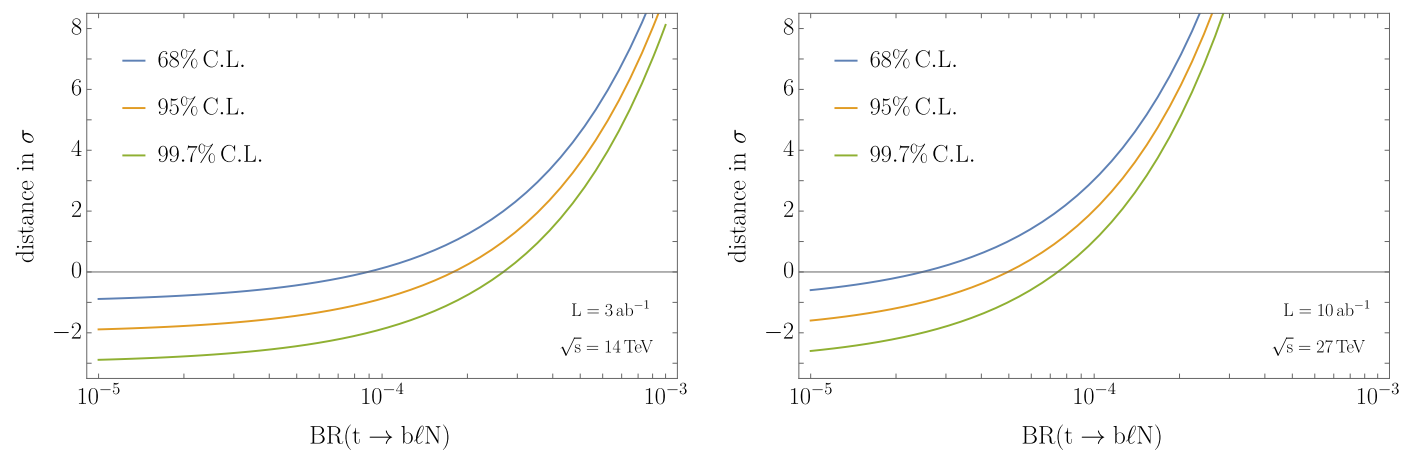

Figure 3. Number of standard deviations between the mean value of the BDT asymmetry in the signal + background and the asymmetry in the SM alone as a function of the exotic top decay mode branching ratio for $\sqrt{s}=14 \mathrm{TeV}$ and $\mathcal{L}=3 \mathrm{ab}^{-1}$ (left) and for $\sqrt{s}=27 \mathrm{TeV}$ and $\mathcal{L}=10 \mathrm{ab}^{-1}$ (right).

expected, $m_{W}^{\ell, 1}$ and $m_{W}^{\ell, 2}$ serve as the best discriminating variables. The other variables do not show significant prowess in the discriminating procedure.

We get a statistical significance of $\sim 13$ for $\operatorname{BR}(t \rightarrow b \ell N) \sim 2 \times 10^{-4}$. However, it drops dramatically as soon as systematic uncertainties are included, due to the very small $S / B \sim 0.41 \%$. The asymmetry between the signal and the background in the BDT variable (see figure 2) can however be used to better control the systematic uncertainties. To this aim, we slide the bins from left to right in the corresponding distribution, and construct the variable $A=\left(N_{\text {right }}-N_{\text {left }}\right) /\left(N_{\text {right }}+N_{\text {left }}\right)$, where $N_{\text {left }}\left(N_{\text {right }}\right)$ denotes the number of events to the left (right) of a chosen bin including the bin itself. Systematic uncertainties cancel in this ratio. The number of standard deviations between the asymmetry in the signal + background and the SM alone is shown in figure 3 as a function of $\mathrm{BR}(t \rightarrow b \ell N)$. It can be seen that branching ratios as low as $\sim 2 \times 10^{-4}$ can be tested at the LHC in the long run. Scaling with the larger $t \bar{t}$ cross section, we also estimate the reach at $\sqrt{s}=27 \mathrm{TeV}$ with $\mathcal{L}=10 \mathrm{ab}^{-1}$, that improves by a factor of $\sim 4$. The projected $95 \%$ C.L. bounds on the 
various couplings entering this rare decay, as described in eq. 2.27, are shown in tables 5 and 7 for the HL-LHC analysis.

In summary, we find that the BDT combined with the asymmetry variable helps in constraining these EFT couplings. More studies are required in this direction and a control over systematics is warranted.

\section{Conclusions}

By using LHC searches for light leptons or taus with missing energy, monojet analyses, and measurements of different pion and tau decay widths, we have singled out the most constrained directions in the $\nu \mathrm{SMEFT}$ and, as a consequence, those others in which new physics can be more likely hiding. (Our results are valid for a cutoff above the EW scale provided $N$ is the $\mathrm{RH}$ component of the SM Dirac neutrino or a new Majorana particle with $0.01 \mathrm{GeV} \lesssim m_{N} \lesssim 0.1 \mathrm{GeV}$.)

In the first category, our limits range from $\sim 380$ (1400) GeV to $\sim 3$ (11) TeV for couplings of order $\sim 1(4 \pi)$; corresponding to leptonic operators with taus and first generation quark-lepton operators, respectively. In the category of unconstrained operators, we find interactions involving heavier quarks, as well as operators that could be better probed at lepton facilities. However, the operators giving genuinely new signals are those providing new top decays. In particular, the operators $\mathcal{O}_{d u N e}, \mathcal{O}_{l d q N}, \mathcal{O}_{l N q d}$ and $\mathcal{O}_{q u N l}$ trigger the process $t \rightarrow b \ell+$ inv, with the missing energy and the lepton not reconstructing a $W$ boson. This not yet explored process is the only sensible signature of these operators. We have worked out a BDT analysis sensitive to this decay channel in semileptonic $t \bar{t}$ production relying mostly on the invariant mass of the lepton and the missing energy; obtained upon requiring the top decay products to reconstruct the top mass.

The BDT variable turns out to be sensibly shifted in the signal with respect to the background. In order to minimise systematic uncertainties, we have based the statistical analysis on an asymmetry built out of the BDT variable. Considering one single lepton family, we have shown that, at the LHC with $\sqrt{s}=14$ (27) TeV and 3 (10) $\mathrm{ab}^{-1}$ of collected luminosity, branching ratios of the top quark into this exotic decay mode as small as $\sim 2 \times 10^{-4}\left(\sim 5 \times 10^{-5}\right)$ can be probed at the $95 \%$ C.L. This translates into a prospective lower bound on $\Lambda \sim 330$ (460) $\mathrm{GeV}$ for $\alpha \sim 1 ; \Lambda \sim 1.2$ (1.6) $\mathrm{TeV}$ for $\alpha \sim 4 \pi$. These numbers can rise up to $\Lambda \sim 1.8(2.5) \mathrm{TeV}$ in the strongly interacting limit if both electrons and muons as well as three RH neutrinos are present.

Finally, let us emphasise that the aforementioned results are also useful to constrain certain operators in the usual SMEFT. Indeed, although SMEFT operators contributing to $t \rightarrow b \ell+$ inv typically also induce quark-charged lepton interactions (because LH neutrinos share $\mathrm{SU}(2)_{L}$ doublet with the charged leptons; and in this respect the $\nu \mathrm{SMEFT}$ is qualitatively different), some directions can be mostly constrained upon exploiting the new top decay we have just studied. For example, let us expand the operator

$$
\begin{aligned}
\mathcal{O} & =\left(\overline{l_{i L}} \gamma^{\mu} l_{i L}\right)\left(\overline{q_{3 L}} \gamma_{\mu} q_{3 L}\right)+\left(\overline{l_{i L}} \gamma^{\mu} \sigma^{I} l_{i L}\right)\left(\overline{q_{3 L}} \gamma_{\mu} \sigma_{I} q_{3 L}\right) \\
& =\left(\overline{\nu_{i L}} \gamma^{\mu} \nu_{i L}\right)\left(\overline{t_{L}} \gamma_{\mu} t_{L}\right)+\left(\overline{\ell_{i L}} \gamma^{\mu} \ell_{i L}\right)\left(\overline{b_{L}} \gamma_{\mu} b_{L}\right)+\left[\left(\overline{\ell_{\boldsymbol{i L}}} \gamma^{\boldsymbol{\mu}} \boldsymbol{\nu}_{\boldsymbol{i} \boldsymbol{L}}\right)\left(\overline{\boldsymbol{t}_{\boldsymbol{L}}} \gamma_{\boldsymbol{\mu}} \boldsymbol{b}_{\boldsymbol{L}}\right)+\text { h.c. }\right]
\end{aligned}
$$


The non-boldface interactions are very hard to probe at hadron colliders. At lepton facilities, one could study the $e^{+} e^{-} \rightarrow b \bar{b}$, but any departure from the SM could be attributed to e.g. RRRR operators, unless the rare top decay is also tagged.

Note added. During the final stages of this work, ref. [44] appeared, in which the bounds on some operators coming from neutrino experiments are derived.

\section{Acknowledgments}

We would like to thank Gurpreet Chahal, Xiao-Dong Ma, Arcadi Santamaria and Jakub Scholtz for helpful discussions. JA is supported by the Spanish MINECO under grant FPA2014-54459-P. SB is supported by a Durham Junior Research Fellowship COFUNDed between Durham University and the European Union under grant agreement number 609412. MC is supported by the Spanish MINECO under the Juan de la Cierva programme and by the Royal Society under the Newton International Fellowship programme. AT acknowledges funding from the European Union's Horizon 2020 research and innovation programme under the Marie Skłodowska-Curie grant agreement No 674896 (ITN Elusives). AT would also like to thank CERN, where part of this work was carried out, for kind hospitality.

Open Access. This article is distributed under the terms of the Creative Commons Attribution License (CC-BY 4.0), which permits any use, distribution and reproduction in any medium, provided the original author(s) and source are credited.

\section{References}

[1] W. Buchmüller and D. Wyler, Effective Lagrangian analysis of new interactions and flavor conservation, Nucl. Phys. B 268 (1986) 621 [INSPIRE].

[2] S. Weinberg, Baryon and lepton nonconserving processes, Phys. Rev. Lett. 43 (1979) 1566 [INSPIRE].

[3] B. Grzadkowski, M. Iskrzynski, M. Misiak and J. Rosiek, Dimension-six terms in the Standard Model Lagrangian, JHEP 10 (2010) 085 [arXiv: 1008.4884] [INSPIRE].

[4] F. del Aguila, S. Bar-Shalom, A. Soni and J. Wudka, Heavy Majorana neutrinos in the effective Lagrangian description: application to hadron colliders, Phys. Lett. B 670 (2009) 399 [arXiv:0806.0876] [INSPIRE].

[5] A. Aparici, K. Kim, A. Santamaria and J. Wudka, Right-handed neutrino magnetic moments, Phys. Rev. D 80 (2009) 013010 [arXiv:0904.3244] [INSPIRE].

[6] S. Bhattacharya and J. Wudka, Dimension-seven operators in the standard model with right handed neutrinos, Phys. Rev. D 94 (2016) 055022 [Erratum ibid. D 95 (2017) 039904] [arXiv: 1505.05264] [INSPIRE].

[7] Y. Liao and X.-D. Ma, Operators up to dimension seven in standard model effective field theory extended with sterile neutrinos, Phys. Rev. D 96 (2017) 015012 [arXiv:1612.04527] [INSPIRE]. 
[8] K. Nakayama, S. Saito, Y. Suwa and J. Yokoyama, Probing reheating temperature of the universe with gravitational wave background, JCAP 06 (2008) 020 [arXiv:0804.1827] [INSPIRE].

[9] Y. Cui, M. Lewicki, D.E. Morrissey and J.D. Wells, Probing the pre-BBN universe with gravitational waves from cosmic strings, JHEP 01 (2019) 081 [arXiv: 1808.08968] [INSPIRE].

[10] Planck collaboration, Planck 2018 results. VI. Cosmological parameters, arXiv: 1807.06209 [INSPIRE].

[11] M. Escudero, Neutrino decoupling beyond the Standard Model: CMB constraints on the dark matter mass with a fast and precise $N_{\mathrm{eff}}$ evaluation, JCAP 02 (2019) 007 [arXiv: 1812.05605] [INSPIRE].

[12] B.C. Canas et al., Updating neutrino magnetic moment constraints, Phys. Lett. B 753 (2016) 191 [arXiv: 1510.01684] [INSPIRE].

[13] O.G. Miranda, D.K. Papoulias, M. Tórtola and J.W.F. Valle, Probing neutrino transition magnetic moments with coherent elastic neutrino-nucleus scattering, arXiv:1905.03750 [INSPIRE].

[14] L. Duarte, J. Peressutti and O.A. Sampayo, Majorana neutrino decay in an effective approach, Phys. Rev. D 92 (2015) 093002 [arXiv: 1508.01588] [INSPIRE].

[15] L. Duarte, I. Romero, J. Peressutti and O.A. Sampayo, Effective Majorana neutrino decay, Eur. Phys. J. C 76 (2016) 453 [arXiv:1603.08052] [INSPIRE].

[16] L. Duarte, G.A. González-Sprinberg and O.A. Sampayo, Majorana neutrinos production at LHeC in an effective approach, Phys. Rev. D 91 (2015) 053007 [arXiv:1412.1433] [INSPIRE].

[17] L. Duarte, J. Peressutti and O.A. Sampayo, Not-that-heavy Majorana neutrino signals at the LHC, J. Phys. G 45 (2018) 025001 [arXiv:1610.03894] [inSPIRE].

[18] A. Caputo, P. Hernández, J. Lopez-Pavon and J. Salvado, The seesaw portal in testable models of neutrino masses, JHEP 06 (2017) 112 [arXiv:1704.08721] [INSPIRE].

[19] J. Alwall et al., The automated computation of tree-level and next-to-leading order differential cross sections and their matching to parton shower simulations, JHEP 07 (2014) 079 [arXiv: 1405.0301] [INSPIRE].

[20] T. Sjöstrand, L. Lönnblad and S. Mrenna, PYTHIA 6.2: Physics and manual, hep-ph/0108264 [INSPIRE].

[21] T. Sjöstrand et al., An introduction to PYTHIA 8.2, Comput. Phys. Commun. 191 (2015) 159 [arXiv: 1410.3012] [INSPIRE].

[22] M. Cacciari, G.P. Salam and G. Soyez, FastJet user manual, Eur. Phys. J. C 72 (2012) 1896 [arXiv:1111.6097] [INSPIRE].

[23] C. Degrande et al., UFO - The Universal FeynRules Output, Comput. Phys. Commun. 183 (2012) 1201 [arXiv: 1108.2040] [INSPIRE].

[24] ATLAS collaboration, Search for a new heavy gauge boson resonance decaying into a lepton and missing transverse momentum in $36 \mathrm{fb}^{-1}$ of pp collisions at $\sqrt{\mathrm{s}}=13 \mathrm{TeV}$ with the ATLAS experiment, Eur. Phys. J. C 78 (2018) 401 [arXiv:1706.04786] [INSPIRE].

[25] A.L. Read, Presentation of search results: the $C L_{s}$ technique, J. Phys. G 28 (2002) 2693 [INSPIRE]. 
[26] CMS collaboration, Search for a $W^{\prime}$ boson decaying to a $\tau$ lepton and a neutrino in proton-proton collisions at $\sqrt{s}=13$ TeV, Phys. Lett. B 792 (2019) 107 [arXiv:1807.11421] [INSPIRE].

[27] Particle Data Group collaboration, Review of particle physics, Phys. Rev. D 98 (2018) 030001 [INSPIRE].

[28] CMS collaboration, Search for new physics in final states with an energetic jet or a hadronically decaying $W$ or $Z$ boson and transverse momentum imbalance at $\sqrt{s}=13$ TeV, Phys. Rev. D 97 (2018) 092005 [arXiv: 1712.02345] [INSPIRE].

[29] M. Carpentier and S. Davidson, Constraints on two-lepton, two quark operators, Eur. Phys. J. C 70 (2010) 1071 [arXiv:1008.0280] [InSPIRE].

[30] J.A. Aguilar-Saavedra, Effective four-fermion operators in top physics: A Roadmap, Nucl. Phys. B 843 (2011) 638 [Erratum ibid. B 851 (2011) 443] [arXiv:1008.3562] [INSPIRE].

[31] G. Durieux, M. Perelló, M. Vos and C. Zhang, Global and optimal probes for the top-quark effective field theory at future lepton colliders, JHEP 10 (2018) 168 [arXiv:1807.02121] [INSPIRE].

[32] G. Durieux, F. Maltoni and C. Zhang, Global approach to top-quark flavor-changing interactions, Phys. Rev. D 91 (2015) 074017 [arXiv:1412.7166] [InSPIRE].

[33] M. Chala, J. Santiago and M. Spannowsky, Constraining four-fermion operators using rare top decays, JHEP 04 (2019) 014 [arXiv: 1809.09624] [INSPIRE].

[34] A. Papaefstathiou and G. Tetlalmatzi-Xolocotzi, Rare top quark decays at a $100 \mathrm{TeV}$ proton-proton collider: $t \rightarrow b W Z$ and $t \rightarrow h c$, Eur. Phys. J. C 78 (2018) 214 [arXiv: 1712.06332] [INSPIRE].

[35] S. Banerjee, M. Chala and M. Spannowsky, Top quark FCNCs in extended Higgs sectors, Eur. Phys. J. C 78 (2018) 683 [arXiv:1806.02836] [INSPIRE].

[36] C.-W. Chiang, U.K. Dey and T. Jha, $t \rightarrow c g$ and $t \rightarrow c Z$ in universal extra-dimensional models, Eur. Phys. J. Plus 134 (2019) 210 [arXiv:1807.01481] [INSPIRE].

[37] R. Jain and C. Kao, Charming top decays with a flavor changing neutral Higgs boson and WW at hadron colliders, Phys. Rev. D 99 (2019) 055036 [arXiv:1901.00157] [INSPIRE].

[38] W. Altmannshofer, B. Maddock and D. Tuckler, Rare top decays as probes of flavorful Higgs bosons, Phys. Rev. D 100 (2019) 015003 [arXiv:1904.10956] [InSPIRE].

[39] R.D. Ball et al., Parton distributions with LHC data, Nucl. Phys. B 867 (2013) 244 [arXiv:1207.1303] [INSPIRE].

[40] M. Czakon and A. Mitov, Top ++ : a program for the calculation of the top-pair cross-section at hadron colliders, Comput. Phys. Commun. 185 (2014) 2930 [arXiv:1112.5675] [INSPIRE].

[41] M. Cacciari, G.P. Salam and G. Soyez, The anti- $k_{t}$ jet clustering algorithm, JHEP 04 (2008) 063 [arXiv:0802.1189] [INSPIRE].

[42] A. Hocker et al., TMVA - Toolkit for Multivariate Data Analysis, physics/0703039 [INSPIRE].

[43] D. Ciupke, Study of BDT training configurations with an application to the $Z / H \rightarrow \tau \tau \rightarrow e e$ analysis, (2012).

[44] I. Bischer and W. Rodejohann, General neutrino interactions from an effective field theory perspective, arXiv:1905.08699 [INSPIRE]. 